\title{
Superficial temporal artery flap for reconstruction of complex facial defects: A new algorithm
}

\author{
Tarek M. Elbanoby ${ }^{1,2}$, Serag M. Zidan ${ }^{1}$, Amr M. Elbatawy ${ }^{1}$, Gaber M. Aly ${ }^{1}$, Khallad Sholkamy ${ }^{1}$ \\ ${ }^{1}$ Department of Plastic and Burn Surgery, Al-Azhar University, Cairo; ${ }^{2}$ Craniofacial Unit, Naser Institute Hospital, Cairo, Egypt
}

Background A variety of island flaps can be based on the superficial temporal artery with variable tissue composition. They can be used for defect reconstruction, cavity resurfacing, facial hair restoration, or contracture release.

Methods Seventy-two patients underwent facial reconstruction using a superficial temporal artery island flap from October 2010 to October 2014. The defects had various etiologies, including trauma, burns, tumors, exposed hardware, and congenital causes. We classified the patients by indication into 5 groups: cavity resurfacing, contracture release, facial hair restoration, skin coverage, and combined. The demographic data of the patients, defect characteristics, operative procedures, postoperative results, and complications were retrospectively documented. The follow-up period ranged from 24 to 54 months.

Results A total of 24 females and 48 males were included in this study. The mean age of the patients was $33.7 \pm 15.6$ years. The flaps were used for contracture release in 13 cases, cavity resurfacing in 10 cases, skin coverage in 17 cases, facial hair restoration in 19 cases, and combined defects in 13 cases. No major complications were reported.

Conclusions Based on our experiences with the use of superficial temporal artery island flaps, we have developed a detailed approach for the optimal management of patients with composite facial defects. The aim of this article is to provide the reader with a systematic algorithm to use for such patients.

Keywords Face / Reconstructive surgical procedures / Algorithms / Hair / Surgical flaps
Correspondence: Tarek M. Elbanoby Department of Plastic and Burn Surgery, Al-Azhar University, 41st Berm eltonsy St., Cairo, Egypt Tel: +20-10-0148-4943 Fax: +20-22-532-6791 E-mail: elbanoby@azhar.edu.eg

This article was presented by the first author at the 16th Congress of the International Society of Craniofacial Surgery (ISCFS) Japan on September 2015, in Tokyo, Japan.

I want to thank Dr. Mahmoud Eldosoky for his contribution with the illustration.

This article contains Supplemental Figs. S1-S4.

Received: 21 Feb 2017 - Revised: 4 Oct 2017 Accepted: 18 Oct 2017

pISSN: 2234-6163 • elSSN: 2234-6171 • https://doi.org/10.5999/aps.2017.00360 • Arch Plast Surg 2018;45:118-127

\section{INTRODUCTION}

Facial defect reconstruction poses several specific challenges for surgeons. Facial defects may result from the surgical extirpation of tumors or vascular anomalies, as well as trauma and burns or their sequelae of scarring and deformities [1]. Moreover, recent changes in wound patterns in Egypt and the increasing prevalence of firearm injuries have resulted in many cases with complex defects. Most of these cases necessitate immediate and delayed reconstructive procedures, as well as revision surgery.
Reconstructive procedures for facial defects vary widely depending on the size and position of the defect, as well as the quality of the surrounding tissue, in addition to the age, general health, and aesthetic sensibilities of the patient [2]. The primary goal that should be considered in facial reconstruction is restoring the anatomical and functional entities of the reconstructed region, while respecting the aesthetic units [3].

The superficial temporal artery and vein are particularly important, as they can be used as a vascular pedicle for vast arrays of fascial (temporoparietal fascia) and cutaneous flaps (frontal 
and temporal skin). Moreover, calvarial bone has been successfully included in fascial flaps based on a superficial temporal vascular pedicle [4].

The utility of these flaps stems from their robust vascularity, ample arc of rotation to reconstruct defects of any part of the face, and the consistent position of the vascular pedicle, as the superficial temporal artery and vein always have a stable anatomical course [5]. Such robust vascularity has enabled surgeons to transfer superficial temporal artery flaps as pedicled or free flaps [6].

In this article, we report our experiences using superficial temporal artery island flaps in facial reconstruction. We have used variable types and tissue compositions of these flaps for facial defect reconstruction, cavity filling, contracture release, and fa- cial hair restoration. We propose a step-by-step detailed algorithm to achieve the appropriate therapeutic goals and to select the proper composition of the flap for each purpose.

\section{METHODS}

\section{Patient population}

Seventy-two patients underwent facial reconstruction using a superficial temporal artery island flap between October 2010 and October 2014. This study included patients treated at $\mathrm{Al}$ Azhar University Hospitals. Patients' demographic data, the defect characteristics, the operative procedure, the postoperative results, and complications were retrospectively documented (Table 1). We classified the patients by indication into 5 groups:

Table 1. Demographic and clinical data of the 72 cases analyzed in this study

\begin{tabular}{|c|c|c|c|c|c|c|}
\hline Characteristic & $\begin{array}{l}\text { Cavity } \\
\text { resurfacing }\end{array}$ & $\begin{array}{l}\text { Contracture } \\
\text { release }\end{array}$ & $\begin{array}{l}\text { Facial hair } \\
\text { restoration }\end{array}$ & Skin coverage & Combined group & Total \\
\hline No. of cases & 10 & 13 & 19 & 17 & 13 & 72 \\
\hline Mean age (yr) & $42.1 \pm 15.1$ & $23 \pm 9.3$ & $25.2 \pm 16.6$ & $45 \pm 16$ & $29 \pm 3.6$ & $33.7 \pm 15.6$ \\
\hline Sex (male:female) & $7: 3$ & $6: 7$ & $14: 5$ & $13: 4$ & $8: 5$ & 48:24 \\
\hline \multicolumn{7}{|l|}{ Defect etiology } \\
\hline Trauma & 5 & 1 & 5 & 7 & 0 & 19 \\
\hline Burn & 0 & 12 & 12 & 0 & 13 & 37 \\
\hline Tumor excision & 5 & 0 & 0 & 9 & 0 & 14 \\
\hline Exposed hardware & 0 & 0 & 0 & 1 & 0 & 1 \\
\hline Congenital & 0 & 0 & 1 & 0 & 0 & 1 \\
\hline \multicolumn{7}{|l|}{ Defect site } \\
\hline Malar region & 0 & 0 & 0 & 3 & 0 & 3 \\
\hline Infraorbial region & 0 & 0 & 0 & 5 & 0 & 5 \\
\hline Nose & 0 & 0 & 0 & 1 & 0 & 1 \\
\hline Palate & 2 & 0 & 0 & 0 & 0 & 2 \\
\hline Eyelid & 0 & 13 & 0 & 8 & 13 & 34 \\
\hline Eyebrow & 0 & 0 & 14 & 0 & 13 & 27 \\
\hline Beard & 0 & 0 & 2 & 0 & 0 & 2 \\
\hline Mustache & 0 & 0 & 3 & 0 & 0 & 3 \\
\hline Orbit & 4 & 0 & 0 & 0 & 0 & 4 \\
\hline Orbit and malar region & 2 & 0 & 0 & 0 & 0 & 2 \\
\hline Orbit with eyelid & 2 & 0 & 0 & 0 & 0 & 2 \\
\hline \multicolumn{7}{|l|}{ Flap composition } \\
\hline Fasciocutaneos & 0 & 13 & 19 & 17 & 13 & 62 \\
\hline Fascial & 4 & 0 & 0 & 0 & 0 & 4 \\
\hline Fasciocutaneos with fascial extention & 4 & 0 & 0 & 0 & 0 & 4 \\
\hline Osteofascial & 2 & 0 & 0 & 0 & 0 & 2 \\
\hline \multicolumn{7}{|l|}{ Mean flap size $\left(\mathrm{cm}^{2}\right)$} \\
\hline Skin paddle & $5.5 \times 2$ & $3.6 \times 0.5$ & $5.6 \times 3$ & $7.4 \times 3.3$ & $1.6 \times 3.4$ & \\
\hline Fascia $^{\text {a) }}$ & $18.8 \times 2.1$ & - & - & - & - & $18.8 \times 2.1$ \\
\hline Bone & $6 \times 3.5$ & - & - & - & - & $6 \times 3.5$ \\
\hline \multicolumn{7}{|l|}{ Complications } \\
\hline Infection & 1 & 0 & 0 & 1 & 0 & 2 \\
\hline Dehiscence & 1 & 0 & 2 & 0 & 1 & 3 \\
\hline Partial flap loss & 0 & 0 & 1 & 0 & 0 & 1 \\
\hline Patchy hair loss & 0 & 0 & 1 & 2 & 1 & 4 \\
\hline Ectropion & 0 & 2 & 0 & 0 & 0 & 2 \\
\hline Lagophthalmos & 0 & 1 & 0 & 0 & 0 & 1 \\
\hline
\end{tabular}


cavity resurfacing, contracture release, facial hair restoration, skin coverage, and combined (in which we performed both hair restoration and contracture release). The follow-up period ranged from 24 to 54 months.

\section{Surgical technique}

At first, the superficial temporal artery and its branches were identified by digital palpation. The skin island was designed either over the lateral aspect of the forehead along the temporal hairline or the temporal area of the scalp, depending on whether the recipient area was to be hairy or non-hairy.

The skin incision began a few millimeters anterior to the tragus and extended upward, following the course of the artery, and ended at the proximal end of the skin island. The superficial temporal artery and its branches were easily identified at this point and exposed. The skin island borders were then incised and flap elevation proceeded from the distal aspect to the proximal aspect. Care was taken to avoid injuring the pedicle while it entered the flap.

A facial extension from the temporoparietal fascia was added to the flap when needed for reconstruction, as in orbital resurfacing. In cases where no skin was necessary, the fascia was harvested without a skin paddle. The dimensions of the harvested fascia were up to $10 \times 15 \mathrm{~cm}$. The plane of flap elevation was deep to the temporoparietal fascia. If bone was to be included, a cuff of fascia and pericranium was preserved at the periphery of the flap and left attached to a segment of the parietal bone. The outer table was harvested using an oscillating right-angled saw and a 4-mm curved osteotome. Fig. 1 shows the anatomy of the superficial temporal artery and the possible island flaps that

\section{Fig. 1. Superficial temporal artery island flap}

(A) Anatomy of the superficial temporal artery. (B) Temporoparietal fascia flap. (C) Superficial temporal artery island hairy flap. (D) Temporoparietal osteofascial flap. (E) Bifurcated superficial temporal artery island flap.

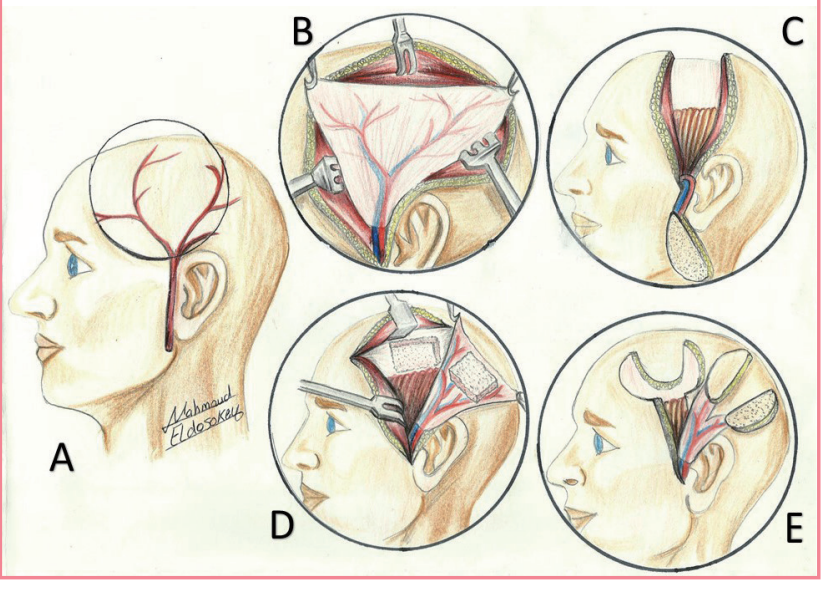

could be harvested based on it.

The flap with its pedicle was then dissected to the level of the tragus to achieve an adequate arc of rotation for the flap. A wide subcutaneous tunnel between the flap and recipient site was dissected, and the flap was transferred and sutured to the skin edges of the defect. In cases of oral cavity resurfacing, the flap was tunneled behind the zygomatic arch. Finally, the fascia was grafted with skin as needed.

When the defect was in the lower face, an extension from the parietal scalp was included after being expanded.

Tips for harvesting the flap include taking care of the hair follicles, and in particular, preserving a thin layer of subcutaneous tissue deep to the hair follicles to avoid donor site alopecia. Incisions should be located away from the arterial course and on the anterior hair line. A wide tunnel is necessary to avoid congestion, and a small amount of scalp subgaleal dissection is needed to close the defect primarily [7].

All human studies were approved by the appropriate Ethics Committee and have therefore been performed according to the ethical standards outlined in the 1964 Declaration of Helsinki and its later amendments.

All patients consented for their photos to be used in scientific journals as well as seminars and clinical presentations.

\section{RESULTS}

The results are summarized in Table 1 . Over a period of 4 years, 72 patients ( 24 females and 48 males) were included in this study. The mean age of the patients was $33.7 \pm 15.6$ years. The defects had etiologies including trauma, burns, tumors, exposed hardware, and congenital causes. All patients underwent facial reconstruction using the superficial temporal artery island flap after a proper preoperative assessment generally and locally. No major complications were reported. The results for each group of patients are presented below.

\section{Cavity resurfacing group}

This group included 10 patients, and we used a superficial temporal artery island flap to resurface the orbital cavity in 8 cases. Fascial flaps were used to resurface the orbital cavity in 4 cases, while fasciocutaneous flaps with a fascial extension were utilized in the other 4 cases, of which a skin paddle was needed to reconstruct the lower eyelid in 2 cases (Supplemental Fig. S1) and the malar region in the other 2 cases. In 2 cases, we reconstructed the palatal cavity using osteofascial flaps (Fig. 2). In both cases, the defect was caused by tumor resection. The bony part was wrapped with fascia and covered with a skin graft for the nasal and oral layers (Supplemental Fig. S2). 
Fig. 2. Palate reconstruction

(A) A 53-year-old female patient presented with a palatal fistula after ablative surgery. (B) After reconstruction with a temporoparietal osteofacial island flap.
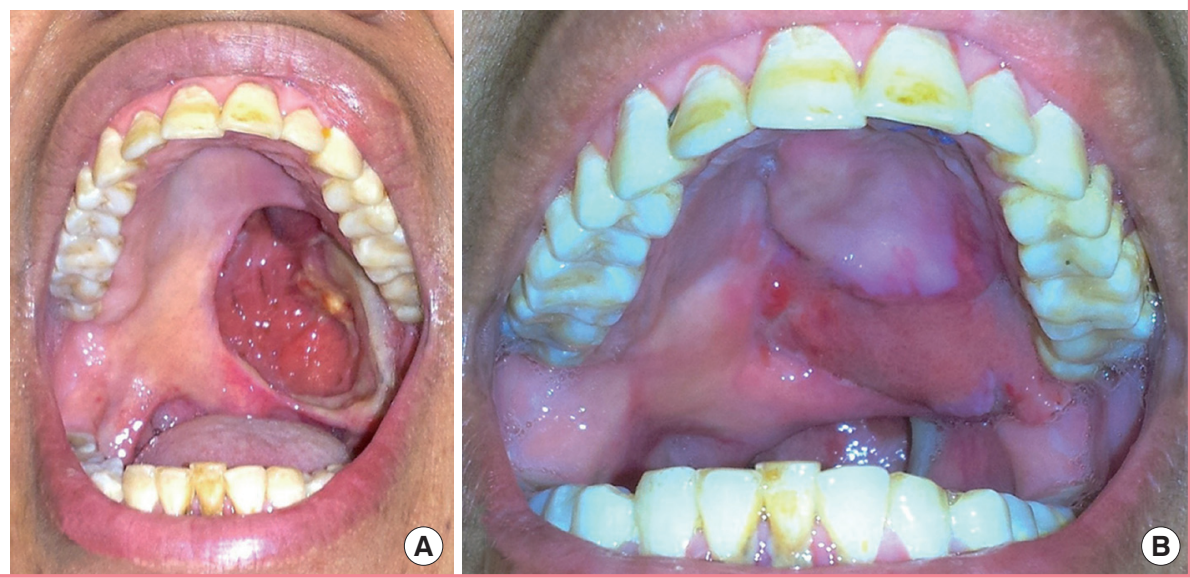

Fig. 3. Restoration of the upper eyelid and eyebrow

(A) A 24-year-old female patient presented with a traumatic degloving injury of the scalp and forehead involving the right eyebrow and upper eyelid. (B) Excessive scarring of the upper eyelid and adjacent forehead area with loss of the eyebrow 45 days after the trauma. (C) After the release of the upper eyelid contracture and restoration of the eyebrow and adjacent forehead area.
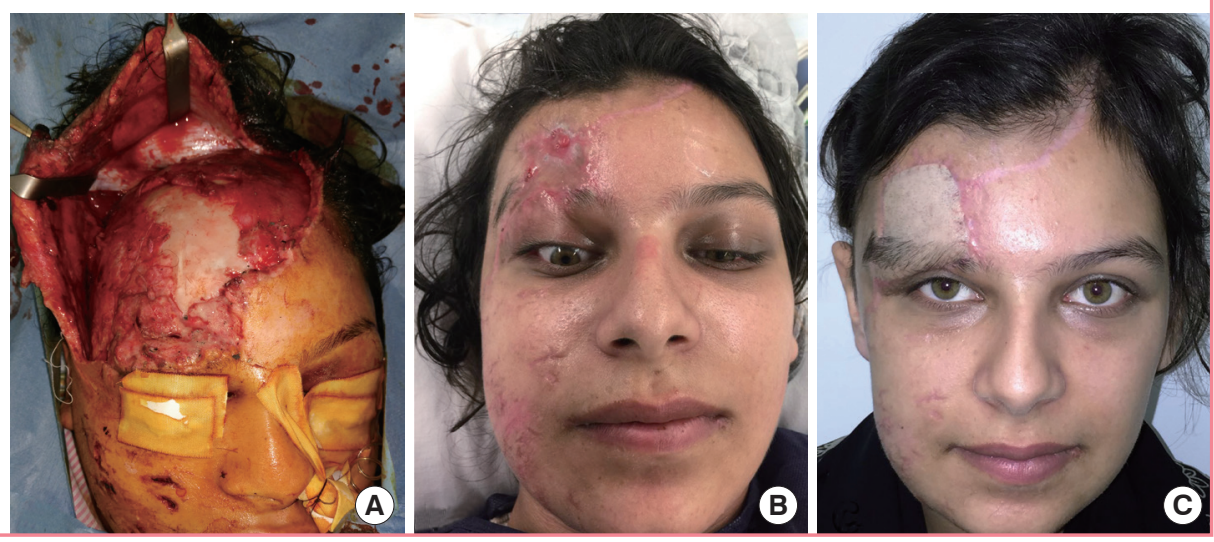

No flap failure was reported in this group, as well as no postoperative infections, apart from 1 case that was managed conservatively. In 1 case of palatal defect, dehiscence occurred 2 weeks postoperatively and was surgically treated by advancement and primary closure. Eight of the donor sites were closed primarily, while two patients needed a skin graft.

\section{Contracture release group}

This group included 13 patients with contracture of the eyelid. This contracture involved an eyelid together with the adjacent aesthetic unit of either the eyebrow or forehead in cases of upper eyelid contractures and the malar region in cases of lower eyelid involvement. The contracture was caused by a burn in all but 1 case, in which it was caused by trauma (Fig. 3). Nine of these cases had recurrent eyelid contracture after release and skin grafting. The release had been done in the upper eyelid in 8 cases and the lower eyelid in 5 cases. Following the release, reconstruction using a superficial temporal artery island flap was performed. Both the upper and lower eyelids were ideally positioned in 10 cases, while postoperative lagophthalmos occurred in 2 cases, and 1 patient complained of scleral show. No ptosis was noted in any cases, and 5 sessions of laser hair ablation were performed in 10 cases. The follow-up period was $36 \pm 6$ months.

\section{Facial hair restoration group}

This group included 19 patients and 20 flaps. Hair restoration was performed in the eyebrow in 14 cases, the mustache in 3 cases, and the beard in 2 cases. Hair loss was a post-burn complication in 12 cases, secondary to trauma in 6 cases, and was congenitally associated with a craniofacial cleft in 1 case. For reconstruction of the beard, a pre-expanded fasciocutanous flap was used for 1 case in the first stage and was then transferred to the lower cheek in the second stage. In the other case, the beard was reconstructed with a pedicled fasciocutanous flap on the ipsilateral side and a free fasciocutanous flap on the contralateral side (Fig. 4). In cases of eyebrow reconstruction, the flap was designed to be similar to the healthy side in its dimensions, the direction of the hair, and transverse length in the ideal position of the eyebrow, which was planned to be refined later by laser hair ablation (Fig. 5). In cases of mustache reconstruction, we 


\section{Fig. 4. Beard hair restoration}

(A) A 30-year-old male patient presented after a gunshot injury with an exposed left ramus of the mandible and loss of the left side of beard. (B) Beard restoration with a 2 fasciocutaneous superficial temporal artery flap, a pedicled ipsilateral superficial temporal artery hairy flap from the same side of the defect and a free superficial temporal artery hairy flap from the contralateral side.
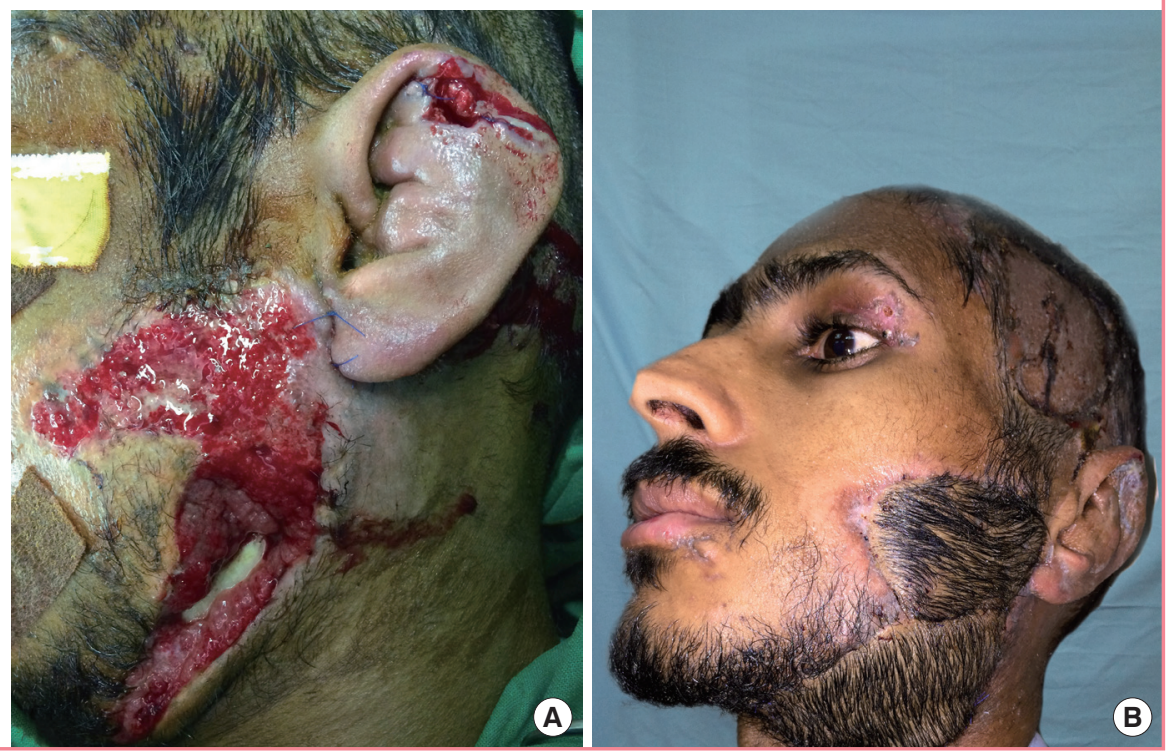

Fig. 5. Eyebrow restoration

(A) A 6-year-old male patient presented with loss of an eyebrow secondary to a craniofacial cleft. (B) Postoperative view 9 months later, after eyebrow restoration with a fasciocutaneous superficial temporal artery island flap.
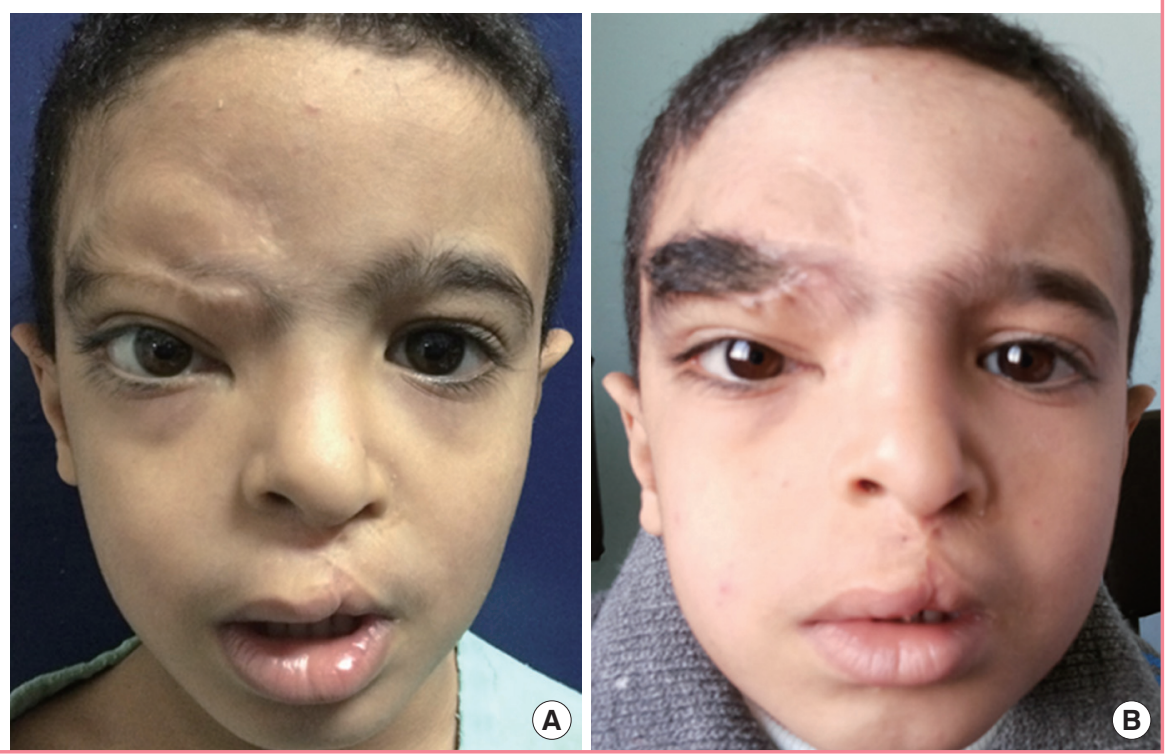

used 2 expanded flaps to simulate the defect. No flap failure or postoperative infections were observed. Surgical refinement, in which the flap was advanced medially, was done in two eyebrow patients to revise the scar 9 months after surgery. Toweling care and laser hair removal were done for nine cases to mimic the normal side.

\section{Skin coverage group}

Seventeen patients underwent surgery to provide skin coverage in various facial regions. Eight patients underwent reconstruction of the eyelid (the lower eyelid in 7 cases and the upper eyelid in 1 case) using a superficial temporal artery island flap. The defects were caused by the excision of basal cell carcinoma in 6 cases and trauma in 2 cases (Supplemental Fig. S3). No complications occurred, no additional operations were performed, and ectropion did not occur during long-term follow-up. The other 9 cases involved reconstruction of the nose in 1 case, the infraorbital region in 5 cases, and the malar region in 3 cases. A superficial temporal artery island flap was used in all cases. Defects in the infraorbital region were caused by tumor resection in three patients, while in a single case, the flap covered an area of exposed mesh in the malar region. The other 5 defects were caused by gunshot injuries. No flap failure occurred in this group. The complications in this cluster were minimal. Minor 
Fig. 6. Bifurcated superficial temporal artery island flap

Separation of the flap under indirect illumination into frontal and temporal flaps, then insetting of the flaps to ensure that they cover both defects. Reprinted from Elbanoby et al. Plast Reconstr Surg Glob Open 2016;4:e748 [7].
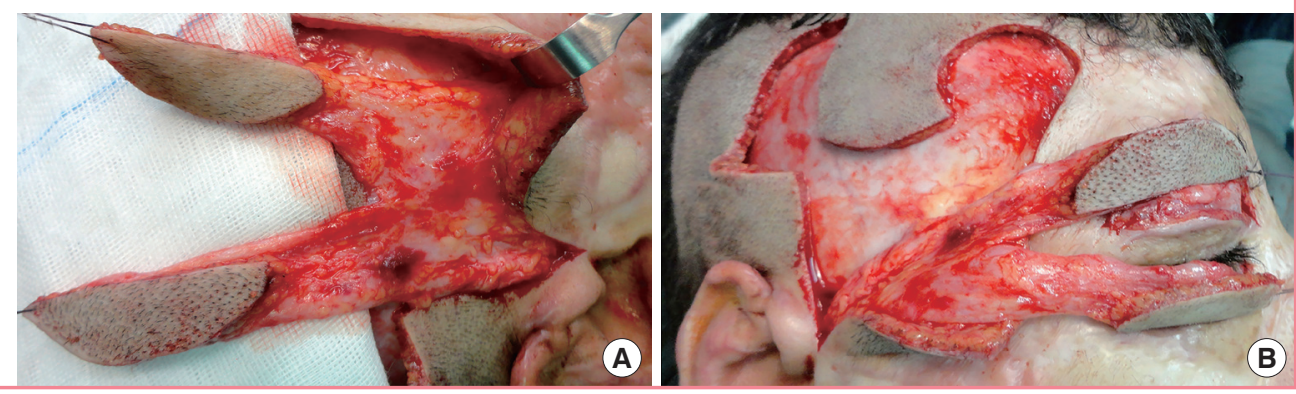

\section{Fig. 7. Periorbital reconstruction}

Male patient, 13 years, the single right eye with post-burn scarring face and neck with severe bilateral periorbital cicatricial scarring. Lower lip ectropion and macrocheilia, late postoperative after bilateral bifurcated superficial temporal artery island flap (BSTIF) and microvascular radial forearm for the neck, and late postoperative with complete eye closure. Reprinted from Elbanoby et al. Plast Reconstr Surg Glob Open 2016;4:e748 [7].
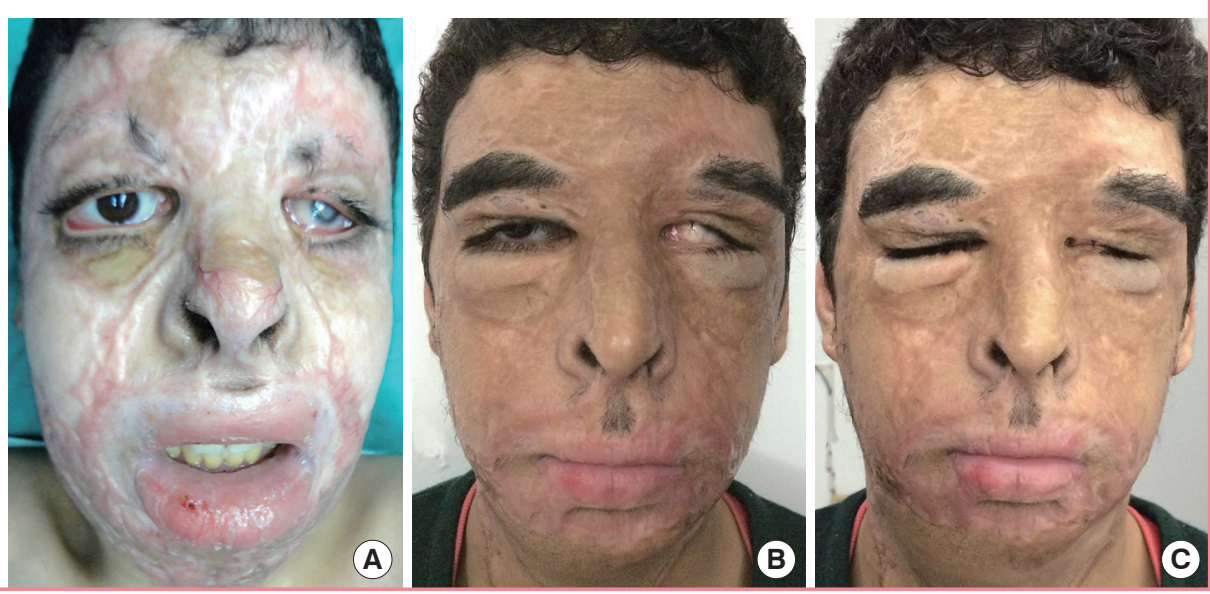

wound infection took place in a single case, and patchy hair loss at the donor site occurred in 2 cases. Laser hair ablation was done in 8 cases in which part of the island flap was hairy. The follow-up period was $36 \pm 12$ months.

\section{Combined group}

This group included 13 cases of post-burn combined alopecia in the eyebrow and contracture of the lower eyelid; both defects were reconstructed with a bifurcated superficial temporal artery island flap, which was innovated by the corresponding author [7]. All patients satisfied the same criteria for periorbital reconstruction using a bifurcated superficial temporal artery island flap to reconstruct the eyebrows and to release ectropion in their upper and lower eyelids. Two patients underwent bilateral flap reconstruction (Fig. 6), and 11 patients underwent unilateral reconstruction; there were no cases of flap failure or postoperative infection. Two eyebrow patients underwent surgical refinement, with 1 receiving scar revision 9 months after surgery and the other having the flap advanced for better positioning and symmetry 6 months after surgery. Laser hair removal was performed in 9 cases to design a new eyebrow mimicking that of the healthy side. The eyelid was observed to have an ideal position in 10 cases; however, in 2 cases, postoperative residual scleral show of 1-2 mm was found, although neither the upper nor lower eyelid required subsequent release after the operation. No complications were noted, apart from 1 case in which hair density was lost in the parietal flap, combined with the loss of the distal $5 \mathrm{~mm}$ of the frontal flap (Fig. 7).

\section{DISCUSSION}

Various reconstructive options are available for repairing facial defects, including skin grafts, local geometric transposition and rotation flaps, axial-pattern regional and distant flaps, and free tissue transfer [8]. However, no ideal solution can be used for every defect, because of color and texture mismatch, technical difficulties, and functional and aesthetic deformities. Therefore, tailoring the reconstructive option according to the site, size, and nature of the defect should be considered in every case [3].

The temporal region is an excellent donor site because of its rich vascular network and its diverse tissues, including skin, fascia, muscle, and calvarial bone. Thus, many flaps can be harvested from this site using 1 or more type of tissue based on the superficial temporal artery and its branches, according to the defect site and nature [9].

Moreover, the superficial temporal vessels provide vast and 
long pedicles and are easy to harvest. The average external diameter of the superficial temporal vessels is more than $2.8 \mathrm{~mm}$. These factors, combined with their consistent position, are why the superficial temporal artery and vein present suitable anatomical characteristics for use as a vascular pedicle in flaps [10].

In this study, we present our experiences with the tailoring of various flaps based on the superficial temporal artery vascular pedicle. At the start of the study, we presented a classification of the patients based on the clinical indication, rather than the type of tissue included in the flap. We used superficial temporal artery island flaps with different compositions as a tool to reconstruct various facial defects.

There is no universal algorithm for facial defect reconstruction in the literature $[11,12]$. In our study, we classified the applica-

\section{Fig. 8. Superficial temporal artery flap algorithm}

Algorithm for the usage of an island temporoparietal fascia flap. LHA, laser hair ablation; STAIF, superficial temporal artery island flap; BSTAIF, bifurcated superficial temporal artery island flap.

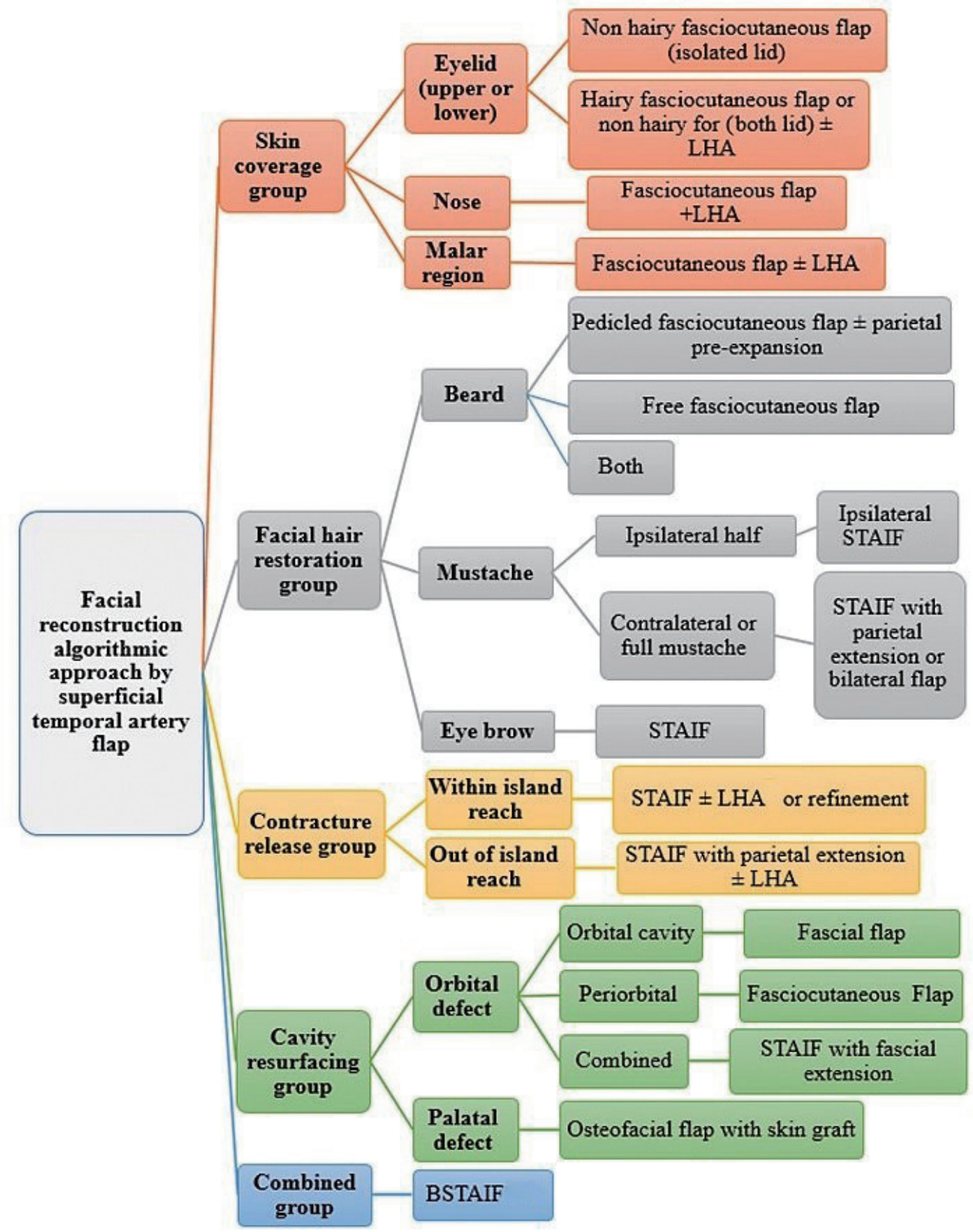


tion of superficial temporal artery flaps to reconstruct a wide range of facial defects, and organized these guidelines as an algorithmic approach (Fig. 8).

An important finding in our case series is the pattern of etiology. The most common etiology was post-burn complications (37 cases), followed by trauma (19 cases). Post-ablative cases accounted for only 14 cases (19.4\%), and only 1 case was observed of both congenital causes and exposed hardware $(<1 \%)$. This is why the mean age of the patients in our study was relatively young ( $33.7 \pm 15.6$ years).

The first group of patients included those who needed resurfacing of the oral and orbital cavities. In cases of oral cavity resurfacing, the temporoparietal osteofascial flap we used to reconstruct the palate achieved its goals, as there was no regurgitation of food or fluids, and the patients' speech became clear and understandable. The superficial temporal artery island flap has been used in oral cavity reconstruction [13,14], and calvarial bone has been included in the flap [5]. Parhiscar et al. [15] reported the usage of temporoparietal osteofascial flaps in 11 cases; 2 of them were used for hard palate reconstruction, with the complete loss of 1 flap. In our series, we had only 1 case of flap dehiscence, which was closed by advancement.

With its proximity to the orbit, reliability, and pliability, pedicled temporoparietal fascia is favorable for the management of various orbital defects. This technique has been described as a single-stage procedure $[16,17]$ or as a 2 -stage procedure with flap prefabrication [18]. In our series, we performed 4 cases of single-stage resurfacing of the orbital cavity using temporoparietal fascia covered with a skin graft, and treated 4 other cases of gunshot injuries to the orbit and adjacent regions. In those cases, we used a temporoparietal fascia flap to resurface the orbital cavity and a skin paddle to reconstruct the adjacent area. Restoration of the orbital socket was achieved, as it could hold and fit an eye prosthesis, with the eyelids retaining the prosthesis in its position.

The second group of patients in this series underwent the release of contracture in 8 upper eyelids and 5 lower eyelids. In general, eyelid contracture is surgically treated. The main alternatives are skin grafts or the transposition of local flaps [19]. However, the use of skin grafts may lead to recurrent contracture; the use of local flaps is preferable whenever possible, since the recurrence rate is substantially lower, but the surrounding local flaps may be not available because of severe scarring. We preferred to use the hairless skin of the temple instead of covering the fascia with skin grafts. Although the operative time was longer, the results were better both functionally and aesthetically due to the stability of the skin and the lower likelihood of recurrence. Ellis et al. [16] reported 2 patients in whom a temporopa- rietal fascia flap was successfully used for lower eyelid and malar reconstruction.

The fasciocutaneous hair-bearing superficial temporal artery island flap has been described for facial hair restoration in the eyebrows [20], mustache (Supplemental Fig. S4) [21], and beard [22]. The third group of patients in this series represents the largest series of facial hair restoration using a superficial temporal artery island flap. We described 2 cases of lower face hair restoration, using a pre-expanded flap in 1 case and a combined ipsilateral pedicle and contralateral free flap in the second case. Gabilondo Zubizarreta and Melendez Baltanas [23] reported 3 cases of beard and mustache reconstruction with a pre-expanded visor flap, and Hafezi et al. [24] likewise published 9 cases of beard and mustache reconstruction with a scalp visor flap without expansion, but we think that this is a complex procedure that needs multiple stages for debulking.

The fourth group of this series included patients in whom the flaps were used to reconstruct defects not specified in other groups. In 17 cases, a superficial temporal artery fasciocutaneous island flap was used with an average skin paddle size of $7.4 \pm 3.3 \mathrm{~cm}^{2}$ to cover skin loss in the eyelid, malar region, infraorbital region, or nose. For the reconstruction of eyelids, we also used the hairless skin paddle of the temple, so the fascia acted only as a carrier of the pedicle. In 3 cases of the reconstruction of other facial regions, we used hairy skin and performed postoperative laser hair ablation. Liu et al. [25] reported the use of prefabricated expanded temporoparietal fascial flaps in cheek and malar reconstruction.

The last group of this series included patients with burned periorbital areas, alopecia in the eyebrows, and ectropion in the upper and lower eyelids with lagophthalmos. The goals of reconstruction in such cases should be to preserve, restore, and maintain function and appearance so that the cornea is protected from ulcers and painful conditions and the patient can return to his or her daily routine in society. To accomplish these goals, surgeons should employ an organized approach to restoring the burned periorbital area, including contracture release with replacement using the uncontracted option and restoration of the hair in the eyebrows [7].

In general, the most common complication related to the island flap is venous congestion, which mainly occurs due to torsion or an improper tunnel. The straight route of the pedicle between the pivot point and the defect site enabled transfer without torsion. Fortunately, no cases of venous congestion occurred in this study, which could be explained by the fact that we preserved the venous plexus in the temporoparietal fascia during the flap dissection, ensured that the pedicle was of adequate length, and performed tunneling of the flap completely under 
vision to avoid torsion of the pedicle and to ensure a tensionfree transfer. Another potential drawback of this procedure is that it typically creates new scars, but these scars were usually hidden. We encountered 3 cases of minimal patchy hair loss.

The limitations of the use of the island superficial temporal artery flap are donor site morbidity, including alopecia and scars, the need for refinements of the flap in another stage, and the arc of rotation, which may limit its use in some defects, although the fact that it is a free flap overcomes this problem.

\section{NOTES}

\section{Conflict of interest}

No potential conflict of interest relevant to this article was reported.

\section{Ethical approval}

The study was approved by the Institutional Review Board of Naser Institute Hospital and performed in accordance with the principles of the Declaration of Helsinki. Written informed consents were obtained.

\section{Patient consent}

The patients provided written informed consent for the publication and the use of their images.

\section{ORCID}

Tarek M. Elbanoby https://orcid.org/0000-0001-8008-0247

\section{REFERENCES}

1. Murillo WL, Fernandez W, Caycedo DJ, et al. Cheek and inferior eyelid reconstruction after skin cancer ablation. Clin Plast Surg 2004;31:49-67.

2. Ausen K, Pavlovic I. Flaps pedicled on the superficial temporal artery and vein in facial reconstruction: a versatile option with a venous pitfall. J Plast Surg Hand Surg 2011;45: 178-87.

3. Mustarde JC. Cheek rotation skin flap to the lower eyelid. In: Strauch B, editor. Grabb's encyclopedia of flaps. Boston: Little Brown; 1990. p.51-6.

4. Davison SP, Mesbahi AN, Clemens MW, et al. Vascularized calvarial bone flaps and midface reconstruction. Plast Reconstr Surg 2008;122:10e-18e.

5. Washio H. Retroauricular-temporal flap. Plast Reconstr Surg 1969;43:162-6.

6. Abul-Hassan HS, von Drasek Ascher G, Acland RD. Surgical anatomy and blood supply of the fascial layers of the temporal region. Plast Reconstr Surg 1986;77:17-28.

7. Elbanoby TM, Elbatawy A, Aly GM, et al. Bifurcated superficial temporal artery island flap for the reconstruction of a periorbital burn: an innovation. Plast Reconstr Surg Glob Open 2016;4:e748.

8. Bradley DT, Murakami CS. Reconstruction of the cheek. In: Baker SR, editor. Local flaps in facial reconstruction. Philadelphia: Mosby/ Elsevier; 2007. p.525-56.

9. Tan O, Atik B, Ergen D. Temporal flap variations for craniofacial reconstruction. Plast Reconstr Surg 2007;119:152e$163 \mathrm{e}$.

10. Pinto F, Magalhaes R, Durazzo M, et al. Galeal flap based on superficial temporal vessels for oral cavity and pharynx reconstruction: an anatomical study. Clinics (Sao Paulo) 2008;63:97-102.

11. Clark N, Birely B, Manson PN, et al. High-energy ballistic and avulsive facial injuries: classification, patterns, and an algorithm for primary reconstruction. Plast Reconstr Surg 1996;98:583-601.

12. Spence RJ. The challenge of reconstruction for severe facial burn deformity. Plast Surg Nurs 2008;28:71-6.

13. Nayak VK, Deschler DG. Pedicled temporoparietal fascial flap reconstruction of select intraoral defects. Laryngoscope 2004;114:1545-8.

14. Beeby MP, Artigot JL, Zuloaga AA. Vascularised temporoparietal fascial flap for closure of an orocutaneous fistula. $\mathrm{Br}$ J Plast Surg 2001;54:275-6.

15. Parhiscar A, Har-El G, Turk JB, et al. Temporoparietal osteofascial flap for head and neck reconstruction.J Oral Maxillofac Surg 2002;60:619-22.

16. Ellis DS, Toth BA, Stewart WB. Temporoparietal fascial flap for orbital and eyelid reconstruction. Plast Reconstr Surg 1992;89:606-12.

17. Lai A, Cheney ML. Temporoparietal fascial flap in orbital reconstruction. Arch Facial Plast Surg 2000;2:196-201.

18. Altindas M, Yucel A, Ozturk G, et al. The prefabricated temporal island flap for eyelid and eye socket reconstruction in total orbital exenteration patients: a new method. Ann Plast Surg 2010;65:177-82.

19. Wainwright DJ. Reconstruction of the peri-orbital region. In: Sood R, Achauer BM, editors. Achauer and Sood's burn surgery: reconstruction and rehabilitation. Philadelphia: Saunders Elsevier; 2006. p.188-99.

20. Motomura H, Muraoka M, Nose K. Eyebrow reconstruction with intermediate hair from the hairline of the forehead on the pedicled temporoparietal fascial flap. Ann Plast Surg 2003;51:314-8.

21. Kim JC, Hadlock T, Varvares MA, et al. Hair-bearing tem- 
poroparietal fascial flap reconstruction of upper lip and scalp defects. Arch Facial Plast Surg 2001;3:170-7.

22. Eser C, Gencel E, Kesiktas E, et al. Outcomes of anatomic reconstruction of gunshot-inflicted lower face defects by free osteoseptocutaneous fibula flap and expanded or nonexpanded temporal scalp flap combination in males. J Craniofac Surg 2016;27:1139-42.

23. Gabilondo Zubizarreta FJ, Melendez Baltanas J. Expanded visor flaps for post-burn beard reconstruction. European Journal of Plastic Surgery 1998;21:399-401.

24. Hafezi F, Naghibzadeh B, Nouhi A. Facial reconstruction using the visor scalp flap. Burns 2002;28:679-83.

25. Liu Y, Jiao P, Tan X, et al. Reconstruction of facial defects using prefabricated expanded flaps carried by temporoparietal fascia flaps. Plast Reconstr Surg 2009; 123:556-61.

\section{Supplementary Material}

Supplemental Fig. S1. Orbital reconstruction

(A) Preoperative: a 25-year-old male patient with extensive periorbital skin loss and a ruptured globe. (B) Postoperative: after bone restoration and soft tissue reconstruction. (C) After debridement and excision of the globe remnant with fixation with microplates all around the orbital rim. (D) Island temporoparietal flap skin island with a temporal fascia extension; the skin island was for the lower eyelid and malar and infraorbital cheek region, while the temporal fascia extension was for eye socket resurfacing with coverage by a splitthickness skin graft. (E) After tunneling of the flap. (F) Postoperative results (https://doi.org/10.5999/aps.2017.00360).

\section{Supplemental Fig. S2. Palate reconstruction}

(A) Harvesting of a temporoparietal osteofacial flap (full-thickness vascularized bone). (B) Insetting of the flap (fascia above the bone as the nasal layer with rigid fixation of bone graft with bony palate). (C) Reinforcement of the fascial extension as the oral layer down the bone graft and coverage of the flap with a split-thickness skin graft. (D) Immediate postoperative result (https://doi.org/10.5999/ aps.2017.00360).

\section{Supplemental Fig. S3. Lower eyelid reconstruction}

(A) A 22-year-old male patient presented after a gunshot injury. (B) lateral view postoperative. (C) Reconstruction of the orbital floor. (D) An island temporoparietal flap was elevated through the coronal approach. (E) Insetting of the flap. (F) Early postoperative picture, before hair ablation (https://doi.org/10.5999/aps.2017.00360).

Supplemental Fig. S4. Eyebrow reconstruction

(A) A 7-year-old male patient with craniofacial cleft and congenital alopecia of the right eyebrow, and the design of the flap and proposed eyebrow. (B) After harvesting of the flap, note that a good tunnel was mandatory to avoid congestion (the white arrows refer to the superficial temporal artery and its two branches). (C) Immediate postoperative photograph with direct closure of the donor site (https://doi.org/10.5999/aps.2017.00360). 